\title{
Radiative emission of solar features in the Ca II K line: comparison of measurements and models ${ }^{\star}$
}

\author{
I. Ermolli ${ }^{1}$, S. Criscuoli ${ }^{1}$, H. Uitenbroek ${ }^{2}$, F. Giorgi ${ }^{1}$, M. P. Rast ${ }^{3}$, and S. K. Solanki ${ }^{4,5}$ \\ 1 INAF-Osservatorio Astronomico di Roma, via Frascati 33, 00040 Monte Porzio Catone, Italy \\ e-mail: ermolli@oaroma.inaf.it \\ 2 National Solar Observatory, Sacramento Peak, PO Box 62, Sunspot, NM 88349, USA \\ ${ }^{3}$ Laboratory for Atmospheric and Space Physics, Department of Astrophysical and Planetary Sciences, University of Colorado, \\ Boulder, CO, 80303, USA \\ 4 Max-Planck-Institut für Sonnensystemforshung, Max-Planck-Strasse 2, 37191 Katlenburg-Lindau, Germany \\ ${ }^{5}$ School of Space Research, Kyung Hee University, Yongin, Gyeonggi 446-71, Korea
}

Received 9 April 2010 / Accepted 18 August 2010

\begin{abstract}
Context. The intensity of the Ca II K resonance line observed with spectrographs and Lyot-type filters has long served as a diagnostic of the solar chromosphere. However, the literature contains a relative lack of photometric measurements of solar features observed at this spectral range.

Aims. We study the radiative emission of various types of solar features, such as quiet Sun, enhanced network, plage, and bright plage regions, identified on filtergrams taken in the Ca II K line.

Methods. We analysed full-disk images obtained with the PSPT, by using three interference filters that sample the Ca II K line with different bandpasses. We studied the dependence of the radiative emission of disk features on the filter bandpass. We also performed a non-local thermal equilibrium (NLTE) spectral synthesis of the Ca II K line integrated over the bandpass of PSPT filters. The synthesis was carried out by utilizing the partial frequency redistribution (PRD) with the most recent set of semi-empirical atmosphere models in the literature and some earlier atmosphere models. As the studied models were computed by assuming the complete redistribution formalism (CRD), we also performed simulations with this approximation for comparison.

Results. We measured the center-to-limb variation of intensity values for various solar features identified on PSPT images and compared the results obtained with those derived from the synthesis. We find that CRD calculations derived using the most recent quiet Sun model, on average, reproduce the measured values of the quiet Sun regions slightly more accurately than PRD computations with the same model. This may reflect that the utilized atmospheric model was computed assuming CRD. Calculations with PRD on earlier quiet Sun model atmospheres reproduce measured quantities with a similar accuracy as to that achieved here by applying CRD to the recent model. We also find that the median contrast values measured for most of the identified bright features, disk positions, and filter bandpasses are, on average, a factor $\approx 1.9$ lower than those derived from PRD simulations performed using the recent bright feature models. The discrepancy between measured and modeled values decreases by $\approx 12 \%$ after taking into account straylight effects on PSPT images. When moving towards the limb, PRD computations display closer agreement with the data than performed in CRD. Moreover, PRD computations on either the most recent or the earlier atmosphere models of bright features reproduce measurements from plage and bright plage regions with a similar accuracy.
\end{abstract}

Key words. Sun: activity - Sun: photosphere - Sun: chromosphere - Sun: faculae, plages

\section{Introduction}

Observations taken with spectrographs and Lyot-type filters in the $\mathrm{Ca}$ II $\mathrm{K}$ resonance line have long served as diagnostics of the solar chromosphere (e.g., Rutten 2007, and references therein) and have been used to determine the temperature structure of semi-empirical atmosphere models (Vernazza et al. 1981; Maltby et al. 1986; Fontenla et al. 1991, 1993). However, except for the quiet Sun (Zirker 1968; White \& Suemoto 1968; Wittmann 1976; Livingston \& Sheeley 2008; Grigoryeva et al. 2009), there is a shortage of measurements in the literature of the radiative emission of solar features observed in the $\mathrm{Ca}$ II $\mathrm{K}$ range at various heliocentric angles.

In addition to providing new data, photometric measurements of disk features in Ca II $\mathrm{K}$ allow us to check the various

\footnotetext{
* Appendix A is only available in electronic form at http: //www . aanda.org
}

semi-empirical models used in spectral line computations (e.g. Rezaei et al. 2008; Reardon et al. 2009; Pietarila et al. 2009) and in the reconstruction of irradiance variations (e.g. Domingo et al. 2009; Harder et al. 2009; Krivova et al. 2009). In particular, models have been presented (Fontenla et al. 2009) that differ from previous sets of models in terms of the physical assumptions used to derive their temperature structure. These models have been adapted in particular for irradiance studies. They were tested against measurements at EUV/FUV, visible, and infrared wavelengths, but comparison with observations in the near UV range was limited. Detailed comparisons between measurements and model predictions in the near UV, including for the Ca II K line, would aid in model refinement.

In this paper, we present a study of the radiative emission of different types of solar features, such as quiet Sun (internetwork), network, enhanced network, plage, and bright plage regions, identified on full-disk observations taken in the spectral range of the $\mathrm{Ca}$ II $\mathrm{K}$ line. The images were obtained with 


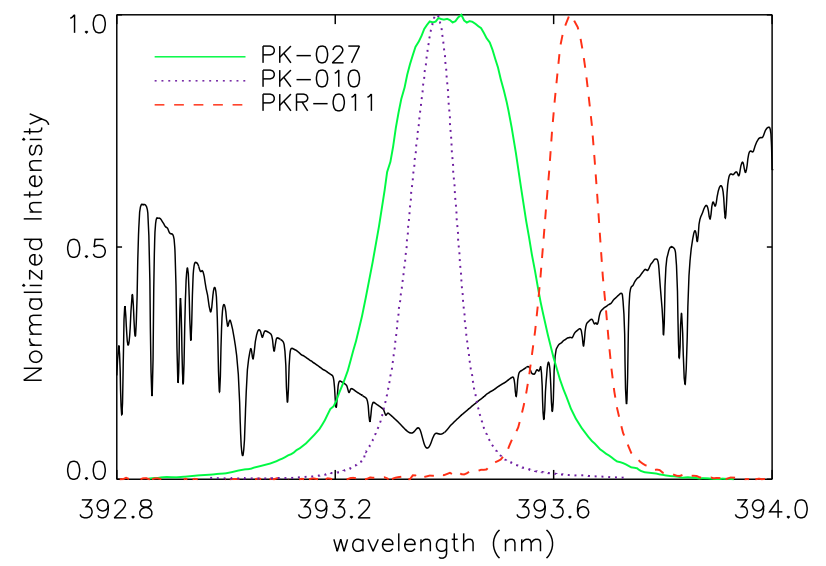

Fig. 1. Transmission profiles of the three PSPT interference filters considered in this study. The three profiles, each normalized to its maximum, are represented with different colors and lines as indicated in the legend. They are superimposed on the solar reference spectrum (black line) provided by Delbouille et al. (1972).

the PSPT (Precision Solar Photometric Telescope), by using three interference filters that sample the Ca II K line with different bandpasses. We studied the dependence of the measured emission on the filter bandpass. We also compared the results obtained with those derived from the numerical synthesis performed for the bandpass of PSPT filters with the RH code (Uitenbroek 2002) utilizing the new models presented by Fontenla et al. (2009) as well as earlier atmosphere models (Vernazza et al. 1981; Fontenla et al. 1993, 2006).

\section{Observations and data reduction}

The PSPT is a $15 \mathrm{~cm}$, low-scattered-light, refracting telescope designed for synoptic photometric solar observations characterized by $0.1 \%$ pixel-to-pixel relative photometric precision (Coulter et al. 1994). Two such telescopes, which differ only in minor hardware characteristics and operational strategies, are operated at Mauna Loa (MLSO-PSPT) and Monte Porzio Catone (Rome-PSPT) Observatories by the High Altitude Observatory (Rast et al. 1999) and Osservatorio Astronomico di Roma (Ermolli et al. 1998), respectively. These telescopes typically acquire full-disk solar images on $2048 \times 2048$ CCD arrays with narrow-band interference filters centred on the blue continuum (409.412 FWHM 0.267 nm), red continuum (607.095 FWHM $0.458 \mathrm{~nm})$, and Ca II K (393.415 FWHM $0.273 \mathrm{~nm}$, hereafter $P K$-027). However, the installation of additional filters on both the telescopes has also allowed the acquisition of images at other spectral ranges. In particular, two interference filters sampling the Ca II K range were added to the MLSO telescope in summer 2007, one at line center (393.379 FWHM $0.103 \mathrm{~nm}$, hereafter $P K-010)$ and the other in the red wing of the line (393.633 FWHM $0.106 \mathrm{~nm}$, hereafter PKR-011). These two filters were operated simultaneously with the broader band $P K-027$ filter. Here we analyse the observations carried out with these three $\mathrm{Ca}$ II K filters, hereafter referred to as the $P K$ filters.

Figure 1 shows the transmission profiles measured for the $P K$ filters superimposed on a reference solar spectrum. The central wavelength and FWHM of these profiles are summarized in Table 2. The filter bandwidths of $P K$ do not allow the features of the Ca II K line center to be resolved. These features, the core, the reversal (emission peaks), and the secondary minima occur within a spectral range less than $0.1 \mathrm{~nm}$ wide.
We analysed 157 sets of observations available from the MLSO PSPT archive $^{1}$ for the period 2007 June 7 to July 31. Each set is composed of the images obtained with the $P K$ filters and one in the red continuum. The red continuum images are, on average, separated in time from the $P K$ ones by $7-8 \mathrm{~min}$.

We separated the data into two samples, corresponding to the highest and the lowest quality images. They contain 37 and 33 sets of images, respectively. Image quality was determined by measuring the width of the solar limb in the $P K-027$ images, which provides an estimate of the atmospheric seeing conditions (Criscuoli et al. 2007; Rast et al. 2008). In particular, images with an average limb width smaller than 4 pixels constitute the highest quality (hereafter referred to as good) images, while the ones with average limb width larger than 5.5 pixels form the set of the worst (hereafter referred to as poor) images. The average and the standard deviation $\sigma$ of limb width values measured for the whole sample of $P K-027$ images are $4.7 \pm 0.8$ pixels; the thresholds introduced above are the values derived from the average $\pm \sigma$.

All the images analysed for our study were pre-processed to apply the correction for gain variations across the CCD device and to compensate for residual large-scale linear gradients affecting the images after CCD calibration (Rast et al. 2008). The images were re-sized and aligned, by using linear interpolation, to a common reference grid on which the solar disk size and orientation are constant.

During the period analysed, the Sun was in a quiet phase. However, eight small active regions appeared on the solar disk, specifically AR 10958 to AR 10965, in addition to some plages and enhanced network regions. To separate the various disk features seen in $P K$ images, we used the image decomposition method described by Fontenla et al. (2009). This method utilizes a threshold scheme derived from the partitioning of intensity histograms constructed from the images as a function of heliocentric angle $(\mu)$. The scheme assigns structure types based on the agreement between the intensity measured at each image pixel and the one defined by the histogram partition. In other words, features are defined by their normalized intensity as a function of $\mu$, and pixels are identified with particular features based on their observed position and intensity.

The decomposition code used in our study assumes seven classes of disk features identified in $P K-027$ and red continuum co-aligned images. Following both the scheme and reference atmospheres proposed by Fontenla et al. (2009), these classes are coded by the letters B, D, F, H, P, S, and R. Features B through $\mathrm{P}$ represent the intensity criterion applied to pixels of $P K-027$ images. They localize average median quiet Sun (internetwork, QS hereafter), network (QS network lanes), enhanced network, plage, and bright plage (or facula) regions, respectively. Features $\mathrm{S}$ and $\mathrm{R}$, which correspond to umbral and penumbral regions, respectively, are identified in red continuum images. The threshold values utilized in $\mathrm{PK}-027$ images are shown in the top panels of Figs. 6 and 7. We note that feature B is called here quiet Sun (QS), but in the threshold scheme localizes more broadly internetwork regions, which likely contain residual magnetism. We also note that the meaning of quiet Sun here is quite distinct from that often used in describing chromospheric or transition region emission, where it is often used to mean the non-coronal hole portion of the Sun. Table 1 summarizes the classes utilized

\footnotetext{
1 Calibrated images from the synoptic observations taken with the two PSPTs over many years are available on-line at http://www. oa-roma.inaf.it/solare and http://lasp.colorado.edu/ pspt_access/.
} 


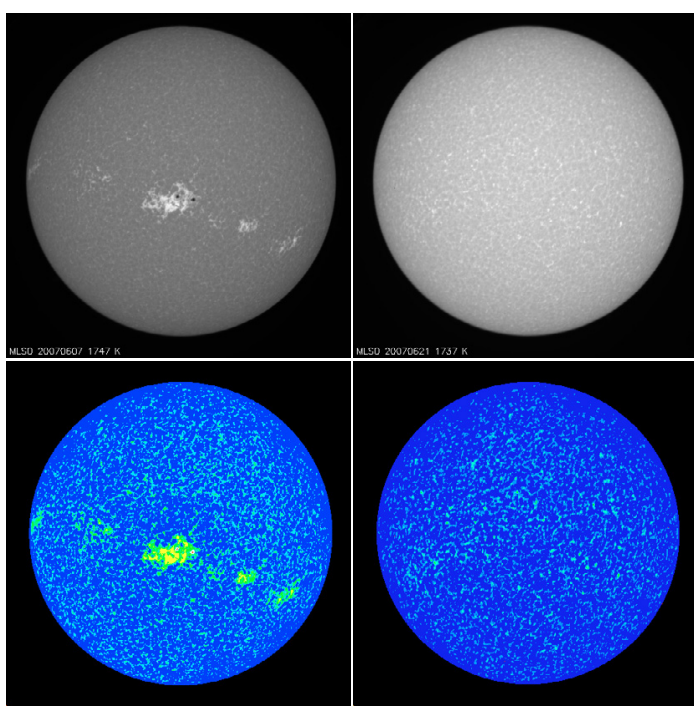

Fig. 2. Example of $P K-027$ (top panels) observations analysed in this study and of the corresponding mask images (bottom panels). The observations were taken on 2007 June 7 (left side) and June 21 (right side). The disk features on mask images are identified by color: blue, light blue, green, and yellow show quiet Sun, network, enhanced network and plage regions, respectively; white and red show penumbral and umbral regions (visible only when enlarging the figure).

Table 1. Identification classes, solar features, and atmosphere models utilized in this study.

\begin{tabular}{lcc}
\hline \hline Class & Solar feature & Atmosphere model \\
\hline $\mathrm{B}$ & quiet Sun & model B, VAL3-C, FAL3-C, FA-06 \\
$\mathrm{D}$ & network & model D, FAL3-F \\
$\mathrm{F}$ & enhanced & model F \\
& network & \\
$\mathrm{H}$ & plage & model H, model F, FAL3-F, FAL3-P \\
$\mathrm{P}$ & bright plage & model P \\
$\mathrm{S}$ & umbra & - \\
$\mathrm{R}$ & penumbra & - \\
\hline
\end{tabular}

Notes. The table summarizes the classes utilized for the identification of the various solar features, the relation between each class and the corresponding solar feature, as well as the relation between solar features and atmosphere models considered for the comparisons presented in the following. Models B to P indicate the set of atmospheres of Fontenla et al. (2009), which constitutes the reference set in this study. Models VAL3-C, FAL3-C, and FA06-C indicate the atmospheres for quiet Sun regions presented by Vernazza et al. (1981), and Fontenla et al. (1993, 2006), respectively. Models FAL3-F and FAL3-P are the atmospheres for plage and bright plage regions presented by Fontenla et al. (1993).

to identify the various solar features, the relation between each class and the corresponding solar feature, as well as the relation between solar features and the various atmosphere models considered in the comparisons presented in the following.

Figure 2 shows examples of features identified in two sets of observations analysed in this study. We measured the radiative emission of the features identified in each image. In particular, we considered the emergent intensity of quiet Sun regions and the contrast of bright features. The latter quantity is defined, for each heliocentric angle, as the ratio $\left(I_{f}-I_{B}\right) / I_{B}$, where $I_{f}$ is the median of intensity values of pixels labeled as feature $f$, and $I_{B}$ is the median intensity of pixels coded as feature $B(\mathrm{QS}$, internetwork). The contrast of features was computed in 50 equal-area annuli centered on the solar disk center. For each bandpass and feature, the center-to-limb variation (CLV, hereafter) of contrast was computed by taking into account the median contrast values measured for the given feature in the 50 annuli of all the images in the specified bandpass. We note that we decomposed $P K-027$ images, and subsequently measured the radiative properties of identified features in PK-027, PKR-011, and PK-010 co-aligned images using this decomposition. This was done to compare measurement and synthesis results assuming that the size of identified features is constant across the various images, i.e., over the atmospheric heights sampled by the $P K$ filters.

\section{Spectral synthesis}

To discuss the results of our measurements and place them in context with results of previous studies, we computed synthetic spectra in the range of the Ca II K line with the $\mathrm{RH}$ radiative code (Uitenbroek 2002). This code performs NLTE radiative transfer modeling by utilizing the partial frequency redistribution formalism (PRD). It has been utilized for spectral synthesis and atmosphere diagnostics at chromospheric heights employing various atmosphere models. For example, Tritschler et al. (2007), Reardon et al. (2009), and Pietarila et al. (2009) utilized RH and earlier models by Fontenla et al. (1993), while Rezaei et al. (2008) and Grigoryeva et al. (2009) employed the sets of Fontenla et al. (2006) and Fontenla et al. (2007).

Our calculations with RH were performed by adopting a 5-level plus continuum atomic model (Uitenbroek 1989, 2001), the atomic data of Shine \& Linsky (1974) with collisional strength values from Meléndez et al. (2007), and the set of atmosphere models presented by Fontenla et al. (2009), hereafter FC09, as well as the earlier models of Vernazza et al. (1981) and Fontenla et al. (1993, 2006), hereafter VAL3, FAL3, and FA06, respectively. Since some of these models were constructed with radiative transfer codes that utilize the complete redistribution approximation (CRD), which is also an option in RH, we computed the Ca II K line profiles with both formalisms for comparison. This was done to evaluate the sensitivity of synthesis results to code approximations.

For each reference model, we calculated the emergent spectrum at 43 wavelengths over the range $392.515 \mathrm{~nm}$ to $394.214 \mathrm{~nm}$ for 13 different viewing angles. For each angle and atmosphere, we weighted the obtained spectrum with the various filter profiles considered in our study and summed up the result over the spectral range of our synthesis. The intensities derived with the code were then compared with those measured in $P K$ images for various disk features. The results obtained are presented in Sect. 4.

As discussed by Shine et al. (1975) and Uitenbroek (2002), we found that the Ca II K line profiles derived from our simulations using the various reference models and adopting either PRD or CRD, differ in terms of the line relative minima. The amplitude of the line emission peaks derived for the various models at disk center, however, is almost unaffected by the adopted redistribution formalism. This is in contrast to the suggestion by Fontenla et al. (2009, their Fig. 7) that the much higher emission peaks in the $\mathrm{H}$ and $\mathrm{K}$ lines they found can be attributed to the lack of inclusion of PRD effects. We note that the difference in emission peak amplitude between CRD and PRD increases considerably towards the limb (Uitenbroek 1989) in any given model, and that this affects the CLV of line-integrated quantities. We also found that the difference between the profiles derived from the two approximations increases according to the brightness of the model disk feature it represents. Comparison of emission peaks obtained with both PRD or CRD also indicates that 


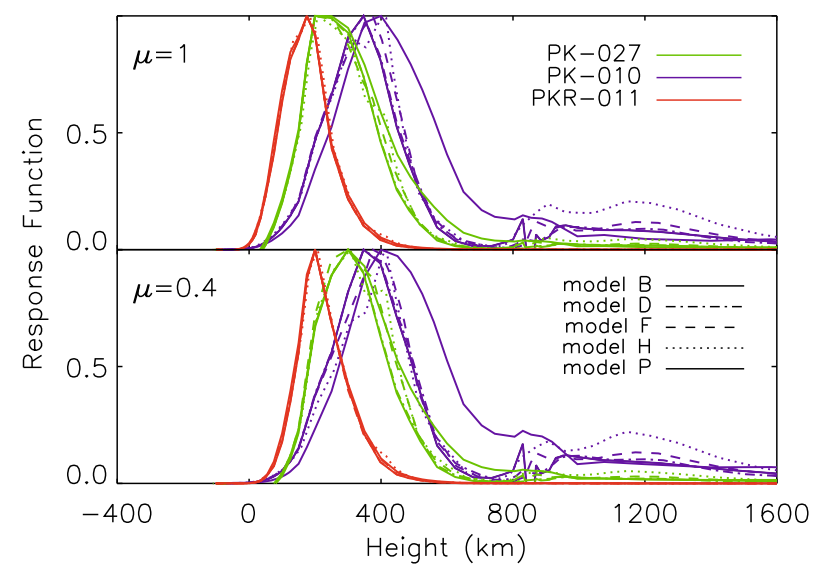

Fig. 3. Intensity response functions to perturbations of temperature for the PSPT filters computed at the disk positions $\mu=1$ (top) and $\mu=0.4$ (bottom). The functions derived for the three filters and various atmosphere models are indicated by different colors and lines as specified in the legend. Each function is normalized to its maximum.

Table 2. Characteristics of the $P K$ filters.

\begin{tabular}{lcccc}
\hline \hline & & $P K-010$ & $P K-027$ & $P K R-011$ \\
\hline$\lambda_{0}$ & $(\mathrm{~nm})$ & 393.379 & 393.415 & 393.633 \\
$\mathrm{BW}$ & $(\mathrm{nm})$ & 0.103 & 0.273 & 0.106 \\
\hline$\mu=1$ & & & & \\
Ave & $(\mathrm{km})$ & $375 \pm 25$ & $200 \pm 5$ & $175 \pm 5$ \\
FW & $(\mathrm{km})$ & $270 \pm 30$ & $260 \pm 15$ & $160 \pm 5$ \\
RF-f & & $0.58-0.82$ & $0.84-0.94$ & $0.996-0.998$ \\
\hline$\mu=0.4$ & & & & \\
Ave & $(\mathrm{km})$ & $380 \pm 30$ & $300 \pm 5$ & $200 \pm 5$ \\
FW & $(\mathrm{km})$ & $270 \pm 35$ & $260 \pm 15$ & $140 \pm 5$ \\
RF-f & & $0.43-0.76$ & $0.75-0.91$ & $0.989-0.995$ \\
\hline
\end{tabular}

Notes. For each bandpass analysed, the table summarizes the central wavelength $\left(\lambda_{0}\right)$ and $F W H M(\mathrm{BW})$ of the filter transmission profile, the average response height (ave), and $F W H M$ of response heights (FW) of the various response functions (RFs) derived from the atmosphere models of Fontenla et al. (2009) at disk positions $\mu=1$ and $\mu=0.4$, and the fraction of RF (RF-f) from atmospheric heights below $500 \mathrm{~km}$ at the same disk positions.

model $\mathrm{P}$ differs from the other models in terms of the smaller value of its micro-turbulent velocity.

Next, we computed the intensity response function (RF) to perturbations of temperature for the $P K$. This function (RFs-PK, hereafter) allows us to infer the atmospheric heights from the intensity measured in $P K$ images more reliably than other methods (Uitenbroek 2006). Moreover, it also indicates the sensitivity of synthesized quantities to atmospheric models, though the CRD and PRD calculations are affected by uncertainties associated with the static and one-dimensional nature of the atmospheres used. We computed the RFs-PK only with PRD because of the advantages that this approach offers for the synthesis of the Ca II K line (Uitenbroek 2002) over the $P K$ range, where the Ca II K dominates all the opacity and emissivity sources. The filter-response functions of the different atmospheres were calculated numerically as described by Uitenbroek (2006), i.e. by perturbing the temperature of an atmosphere at each height, evaluating the emergent intensity integrated over the filter bandpass, and comparing the perturbed filter intensities with those of the un-perturbed atmosphere.

Figure 3 shows the RFs-PK derived for the various atmosphere models at the disk positions $\mu=1$ (top) and $\mu=0.4$

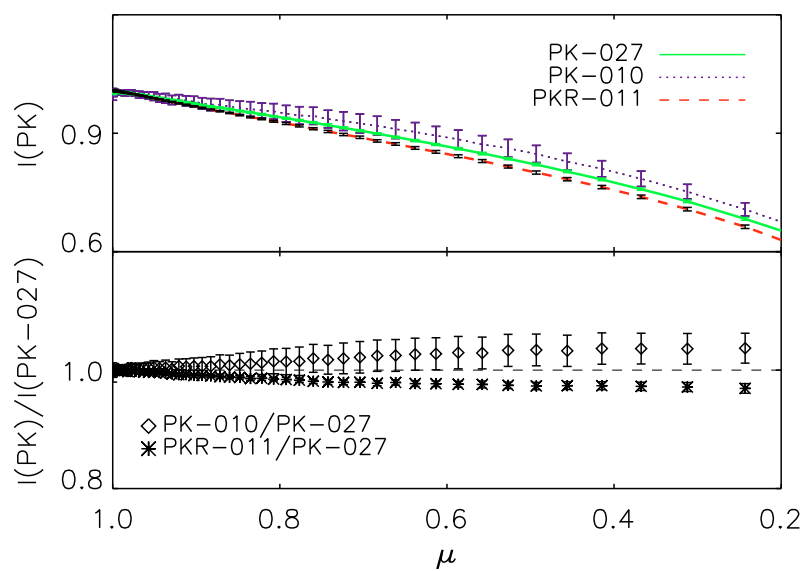

Fig. 4. Top: quiet Sun limb darkening measured in $P K-027$ (green, solid line), $P K-010$ (violet, dotted line), and PKR-011 (red, dashed line) good images. Intensity values are normalized to the median of values measured at the disk center $(\mu \geq 0.95)$. The error bars show the dispersion of measured values. Bottom: ratio of the median of quiet Sun limb darkening measured in $P K-010$ to $P K-027$ (diamond) images, and in $P K R-011$ to $P K-027$ images (asterisk). The error bars show the dispersion of values from error propagation. The dashed line would represent perfect agreement between the two compared sets.

(bottom). These RFs-PK indicate that the $P K$ sample quite a wide range of atmospheric heights, and that the radiative signals measured with these filters are dominated by the wings of the Ca II K line, which form at heights below $500 \mathrm{~km}$. We found that $P K-010$ samples the highest atmospheric regions, while $P K R-011$ and $P K-027$ sample middle and upper photospheric heights, respectively. In particular, $P K R-011$ has the sharpest RF and provides the cleanest photospheric signal. These properties follow from the filter widths and positions.

All RFs-PK display a smooth variation with atmosphere height, except for the ones derived for model B (representative of QS), which show a sharp variation at a height of $\approx 850 \mathrm{~km}$ produced by the temperature profile of this model above the temperature minimum layers. The RF derived for $P K-010$ and $P K-027$ also have a long tail extending to the middle and upper chromospheric heights exceeding $1000 \mathrm{~km}$. However, the response of $P K-027$ is $\approx 3$ times lower than that of $P K-010$ at both heights of $800 \mathrm{~km}$ and $1000 \mathrm{~km}$, for a vertical line of sight and the reference atmosphere model $\mathrm{P}$ (bright plage). In addition, while the RFs-PK derived for models B (QS) to model H (plage) exhibit similar variations with height, the RF-PK computed for model $\mathrm{P}$ (bright plage) is $\approx 3$ times higher than the RF-PK obtained for model $\mathrm{H}$ (plage) at a height of $800 \mathrm{~km}$, but it is $\approx 3$ times lower than the RF for model $\mathrm{H}$ (plage) at $1000 \mathrm{~km}$ height.

Table 2 summarizes the average and FWHM of the response heights of the RFs-PK for the entire set of atmosphere models. We found that these quantities are only slightly sensitive to the choice of atmosphere models and filter profiles. Among the various RFs-PK, the most sensitive to chromospheric conditions are the ones derived for model $\mathrm{P}$ and $P K-010$. We also found that the average response height increases from $1 \%$ to $40 \%$ when moving toward the limb for $P K-027$ and $P K-010$.

\section{Results}

Figure 4 (top panel) shows the CLV of intensity values measured for QS identified in $P K$ images. In particular, the CLV computed from the $P K-010$ images is less steep than the one found in both $P K R-011$ and $P K-027$ observations. Moreover, on average, more 
I. Ermolli et al.: Radiative emission of solar features in the Ca II K line

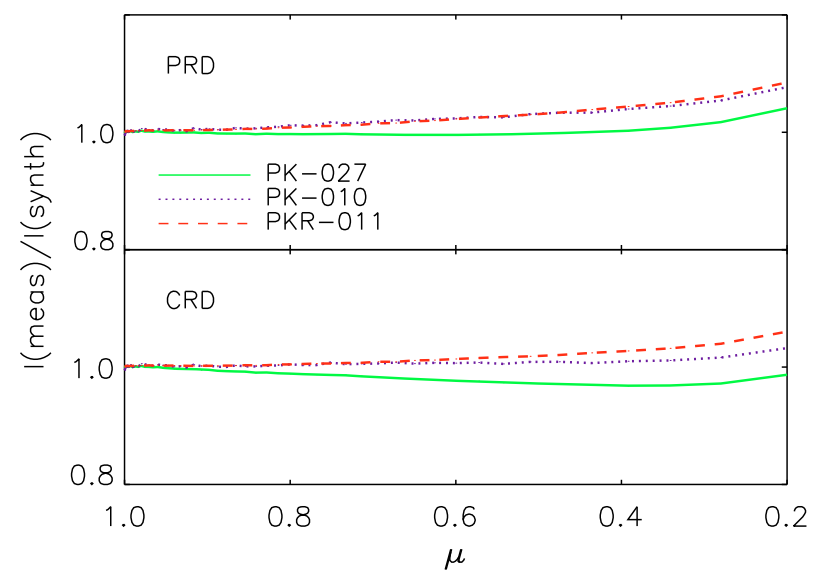

Fig. 5. Ratio between the median of quiet Sun limb darkening measured in PSPT images and the quiet Sun limb darkening derived from the synthesis performed on model B by FC09 for the $P K-027$ (green, solid line), $P K-010$ (violet, dotted line), and PKR-011 (red, dashed line) filters by assuming either the PRD (top panel) or CRD (bottom panel) formalism.

poorly resolved images from the poor quality set lead to the CLV being larger than the median CLV measured for good quality images, although differences are within the deviation of values measured on good images. These findings are in agreement with results obtained for different Ca II K data sets by Livingston \& Sheeley (2008) and Criscuoli \& Ermolli (2008).

The dependence of the intensity CLV on both filter bandwidth and central wavelength is, however, small. We found that the average and the standard deviation of the ratio of the values measured on $P K-010$ to $P K-027$ images are $1.013 \pm 0.013$, for all the disk positions with $\mu \geq 0.2$ (Fig. 4 , bottom panel). The same quantities derived for the ratio of the values measured on $P K R$-011 to $P K$-027 images are $0.99 \pm 0.01$. We note that these averages are strongly weighted by the values obtained at positions close to disk center, because of the larger number of pixels corresponding to large $\mu$.

Comparing the median CLV measured in $P K$ images with the CLV derived from line synthesis, we find that the measured CLVs are generally consistent with the results of the spectral synthesis performed on the reference atmosphere (model B) utilized in this study. In particular, Fig. 5 (top panel) shows this comparison for results derived with PRD. The differences between measured and modeled values increase towards the limb, reaching between $4 \%$ and $8 \%$ depending on the bandpass, with the measured limb values always brighter than model predictions. The average and the standard deviation of the ratio of the measured to modeled values for all the disk positions with $\mu \geq 0.2$ are $0.999 \pm 0.003,1.015 \pm 0.015$, and $1.013 \pm 0.016$ for the $P K-027, P K-010$, and $P K R-011$ images, respectively. The same quantities evaluated for poor quality images are within the deviation of values measured in high quality data. These results indicate that the intensity thresholds utilized in this study for the identification of QS are in fairly good agreement with those obtained with PRD in the reference QS atmosphere (model B) of FC09. Figure 5 (bottom panel) also shows that the agreement between measurements and synthesis results slightly improves by taking into account the results from CRD computations, in line with the increase in emission of line emission peaks towards the limb for profiles calculated with this formalism. We found that the maximum difference between measured and modeled values is $\simeq 5 \%$. The average and standard deviation of the ratio of measured to modeled values obtained with CRD over all disk
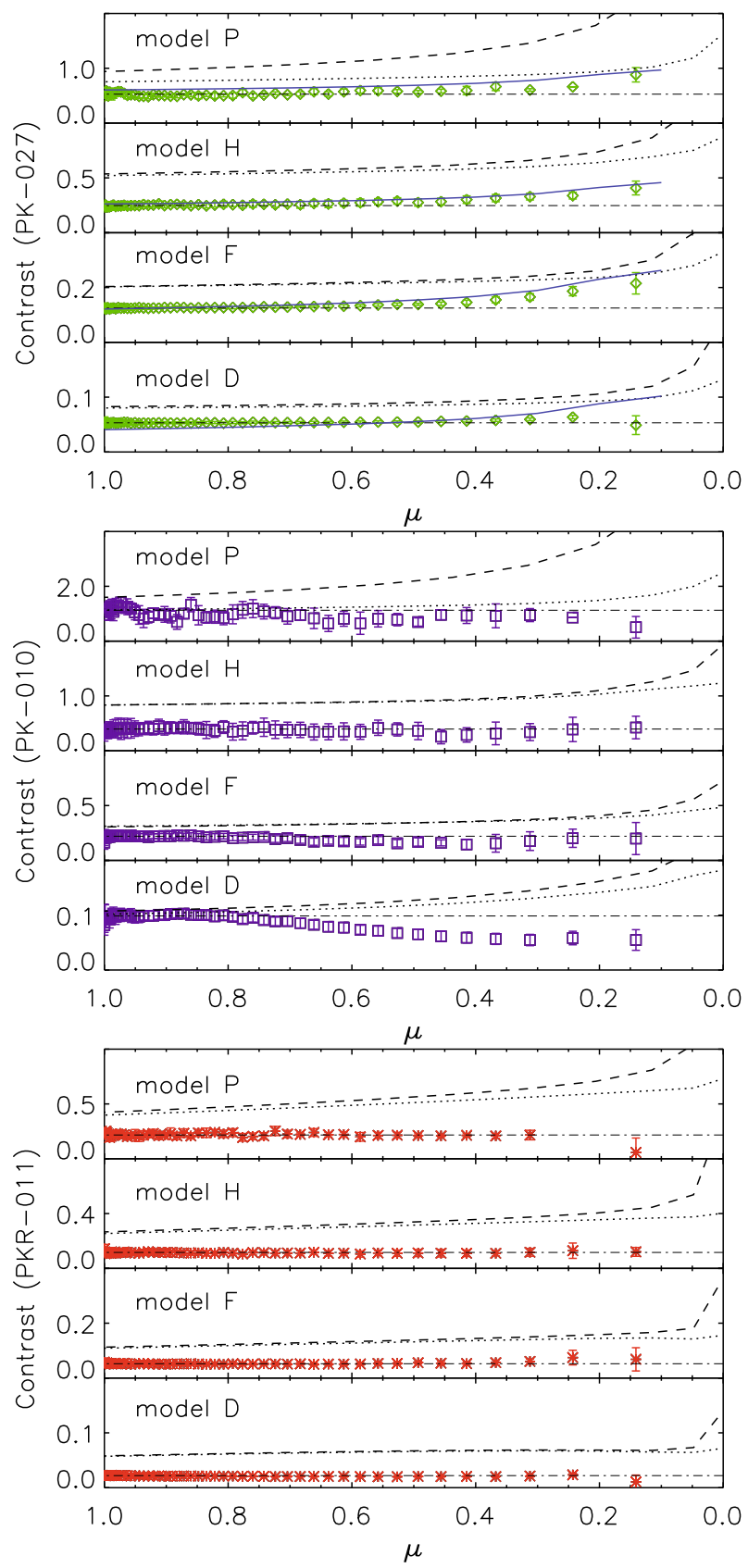

Fig. 6. CLV of median contrast values measured (symbols) for various disk features identified in $P K-027$ (top panel), $P K$-010 (middle panel), and PKR-011 (bottom panel) images. For each bandpass, the various sub-panels show measurement results for solar features ordered by decreasing contrast, i.e. from the top panel results for bright plage, plage, enhanced network, and network regions, respectively. The error bars represent the standard deviation of measurements. For each disk feature and bandpass, the dot-dashed line indicates the average of values measured at disk positions $\mu \geq 0.9$, while dotted and dashed lines show the respective CLV derived from RH calculations with PRD and CRD and the reference model corresponding to the given feature, also indicated in the legend. In the top panel, the solid blue lines show the threshold values applied to the feature identification.

positions with $\mu \geq 0.2$ are $0.99 \pm 0.01,1.006 \pm 0.005$, and $1.01 \pm 0.01$ for the $P K-027, P K-010$, and $P K R-011$ images, respectively. We discuss these results in Sect. 5.

We now consider the contrast measured for the various bright features identified in the $P K$ images. Figure 6 shows that the measured values depend on the type of solar feature, disk 
A\&A 523, A55 (2010)
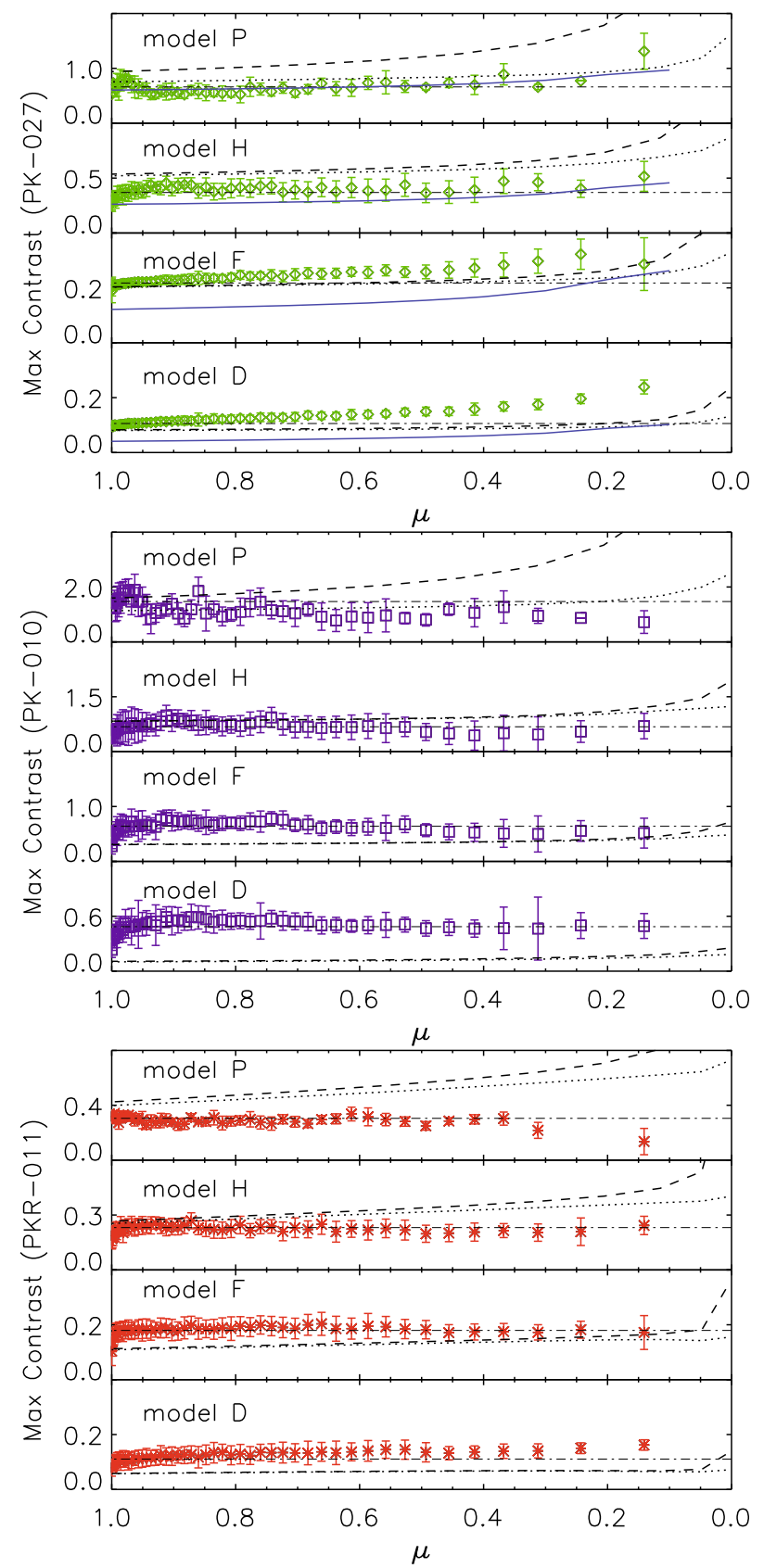

Fig. 7. CLV of maximum contrast values measured (symbols) for various disk features identified in $P K-027$ (top panel), $P K$-010 (middle panel), and PKR-011 (bottom panel) images. The error bars represent the standard deviation of measurements. Legend as in Fig. 6.

position, and filter bandpass. In particular, contrast values measured for each feature at all disk positions in both $P K-010$ and $P K-027$ images are higher than those derived from $P K R-011 \mathrm{ob}-$ servations. This fits in with the well-known observational evidence that the contrast of bright features is greatest at the line core. However, the contrast values measured in the $P K-010 \mathrm{im}-$ ages, for the disk positions with $\mu \leq 0.5$, overlap those obtained for the $P K-027$ observations, which sample more extensively the Ca II K line wings.

We found that the median of the measured contrast values, for a given feature over each $\mu$ range, increases towards the limb for all the features identified in the $P K-027$ images (by $3 \%$ for the network, by up to $24 \%$ for the plage). The same quantities evaluated for all the features identified in PKR-011 observations appear to be constant across the disk, within the scatter of measurements. This is also true for the results obtained in the $P K-010$ images for plage and bright plage features (associated with reference models $\mathrm{P}$ and $\mathrm{H}$, respectively). On the other hand, the network and enhanced network contrast measured in the same images (associated with reference models D and $\mathrm{F}$, respectively) show a clear monotonic decrease toward the limb. This decrease is likely due to both the limited spatial resolution of analysed images and a foreshortening near the limb. We find that the maximum value of contrasts measured for the same features and bandpass over each $\mu$ range is constant across the disk, within the scatter of the measurement uncertainty. Figure 7 shows the CLVs of maximum contrast values measured for the various disk features and bandpasses. These results agrees qualitatively with published CLV measurements of bright features from full-disk continuum observations (Ermolli et al. 2007; Criscuoli \& Ermolli 2008, and references therein).

Figures 6 and 7 also compare the measured and synthetic contrasts of models D, F, H, and P. Figure 6 (all panels) shows that the median of the contrast values measured at the disk center are, on average, a factor $\approx 1.7$ lower than those derived from PRD using the corresponding atmospheric models; the discrepancy increases for plage regions (associated with model $\mathrm{H}$ ) identified in all the images and for all the features in PKR-011. Figure 6 (top and bottom panels) also indicates that the median contrast values deduced from $P K-027$ and $P K R$-011 images are in general poorer agreement with model predictions than the $P K-010$ data.

We also find that the values obtained with CRD for the models $\mathrm{D}$ to $\mathrm{H}$, which are representative of network to plage regions, at disk center, on average, are only $<2 \%$ higher than those obtained with PRD on the same models, but the same quantity evaluated for model $\mathrm{P}$, associated with bright plages, is $\approx 29 \%$, $20 \%$, and $6 \%$ higher in $P K-010, P K-027$, and $P K R-011$ images, respectively. When moving toward the limb, the discrepancy between measured and modeled values slightly decreases for PRD computations, while it increases for CRD calculations. We find that the maximum contrast values measured for most of the solar features, filter bandpasses, and disk positions agree in general more closely with the outcomes of the spectral synthesis than the median contrast measurements. In particular, Fig. 7 (top and middle panels) shows that the CLV of the maximum contrast values measured for plage and bright plage features identified in $P K-010$ images is very satisfactorily reproduced by the CLV of contrast values obtained with PRD for the corresponding reference models $\mathrm{H}$ and $\mathrm{P}$, respectively. This is also true for the same features identified in $P K-027$ images, and for plages in PKR-011 observations, but with lower fidelity.

\section{Discussion}

The PSPT observations analysed in this study are characterized by high photometric precision (Rast et al. 2008; Goldbaum et al. 2009) and a nearly simultaneous sampling of the solar atmosphere in various spectral bands. However, they have only moderate spatial resolution and miss the small-scale chromospheric structure found by high-resolution observations (e.g. Wöger et al. 2006; Rezaei et al. 2008; Cauzzi et al. 2008; Scharmer et al. 2008). Thus, PSPT images are ideal for studying largescale phenomena in the solar atmosphere, as in the present study, in which temporally and spatially averaged intensity measurements are compared with atmosphere models, but are less suited to study of the local dynamics, which tend to be averaged at these pixel resolutions. A potential weakness of our study is that 
I. Ermolli et al.: Radiative emission of solar features in the Ca II K line

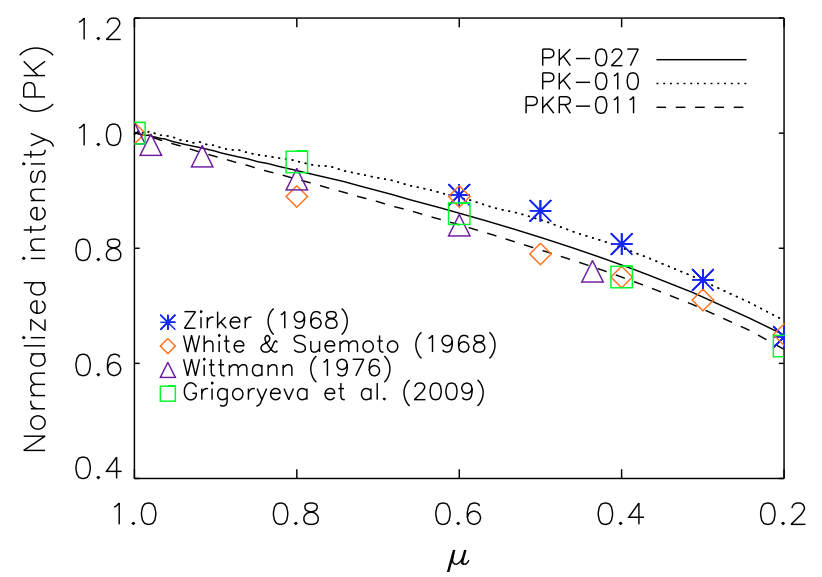

Fig. 8. Comparison between intensity CLV of quiet Sun regions identified in $P K$ images (lines, as described in the legend) and published in the literature (symbols, as described in the legend) from observations taken with spectrographs at the Ca II K line core. Details are given in Sect. 5 .

$P K$ profiles are up to about ten times wider than the bandpasses typically used for Ca II K observations, e.g. of Lyot-type filters and spectrographs (e.g. Kentischer et al. 2008; Ermolli et al. 2009), making quantitative comparison with results from narrower band observations difficult. On the other hand, we note that the bandpass of the $P K-010$ images is similar to that of the Ca II K high-resolution observations from the Swedish Solar Telescope (Scharmer et al. 2003) analysed e.g. by Pietarila et al. (2009), while the bandwidth of $P K-027$ data is close to that of the Ca II H high-resolution images, from the SOT/BFI instrument (Tsuneta et al. 2008) onboard the Hinode spacecraft, studied e.g. by Lawrence \& Cadavid (2010).

To assess the importance of the filter width, we compare our measurements with results available in the literature for observations of the QS taken with spectrographs at the Ca II K line core, i.e. close to the spectral range sampled with the $P K$, but with a narrower bandwidth than that of $P K$. Figure 8 shows that our CLV measurements from $P K-010$ images are in striking agreement with the results presented by Zirker (1968). Moreover, our measurements from $P K-027$ and $P K R-011$ images, i.e. those obtained at the line core with the widest bandpass and in the red line wing, reproduce the results published by White \& Suemoto (1968), Wittmann (1976), and Grigoryeva et al. (2009) fairly well. Thus, the intensity CLV of QS appears to be relatively insensitive to the exact width or central wavelength of the $\mathrm{Ca}$ II K filter. However, it also implies that the measurements by White \& Suemoto (1968), Wittmann (1976), and Grigoryeva et al. (2009) were derived from data obtained in different observational conditions (e.g. spectral sampling, spatial resolution, seeing) and after different types of processing (e.g. data calibration, straylight compensation) than in Zirker (1968).

The close agreement found between our measurements and published results indicates that any systematic errors affecting our QS measurements, originating in the data analysed or caused by image processing applied, do not exceed the scatter in previous measurements. We expect that this also holds for the measurements of the bright features identified at the disk center. However, at the limb, our measurements do not account for the dependence of feature size on both atmosphere height and disk position.

To investigate the effects of feature misclassification on our measurements, we applied a second independent decomposition method to the data, specifically that developed by Nesme-Ribes et al. (1996). A direct comparison of the results obtained with the two methods is hampered by the different number of feature classes. However, the results obtained from this test support those presented in Sect. 4. The contrast values measured for the features identified by the test-processing are, on average, lower than those presented in Sect. 4 and in poorer agreement with the results derived from the synthesis at all disk positions.

In the present study, we compared PSPT measurements with the outcome of spectral synthesis performed with a wellvalidated code on the FC09 atmosphere models. We are aware that none of these models can realistically reproduce the very inhomogeneous atmosphere depicted by high-resolution observations. Some spatial and temporal scales of atmosphere patterns displayed by high-resolution observations, as well as some amplitudes of temperature fluctuations, can be partly reproduced only by hydrodynamic models (e.g. Carlsson \& Stein 1995, 1997; Wedemeyer-Böhm et al. 2004; Hansteen et al. 2007; Leenaarts et al. 2009). However, other temporally and spatially averaged properties of the solar atmosphere deduced from observations, e.g. the ones discussed by Fontenla et al. (2009), are reproduced fairly precisely by the static and one-dimensional models, such as those considered in our study. As discussed by Rezaei et al. (2008), these models can be employed to derive a number of diagnostics (e.g. response functions to temperature perturbations) and to represent temperature and density variations as a function of height in the solar atmosphere, at least across the spectral range and at the temporal and spatial resolutions of observations analysed for our study.

We find that the agreement between measured and modeled results for the intensity CLV of QS varies with the redistribution formalism used in the spectral synthesis. In particular and somewhat surprisingly, the measurements are, on average, in slightly closer agreement with CRD computations than the PRD calculations. This may be because the atmosphere models utilized in this study were computed assuming CRD (Fontenla et al. 2009). If so, CRD, which results in enhanced emission towards the limb, compensates for the steep CLV that would result from the low density chromospheric temperature if the more physically realistic formulation of PRD were included. Therefore, the reference model needs to be modified to fit the data using the PRD formulation.

To investigate the effects of adopting different reference models on the results obtained, we compared measured values for the various disk features and bandpasses with the outcomes of the synthesis performed with PRD on earlier sets of semi-empirical models. In particular, we analysed the VAL3 and FAL3 series, and their subsequent upgrade FA06. Figure 9 shows the ratio of the CLV of median QS intensity values measured in $P K$ images to the CLV derived from PRD computations for the quiet Sun models of the series indicated above. For all bandpasses, the intensity measurements are best reproduced by the synthesis performed on the QS model of FAL3 series (FAL3-C). For some bandpasses, synthesis results for the QS models of both VAL3 and FA06 series (VAL3-C and FA06-C, respectively) reproduce measured values more closely than achieved with the QS atmosphere (model B) of FC09. In addition, computations using the bright feature models of the FAL3 set (FAL3-F and FAL3-P) reproduce the contrast measurements for plage and bright plage features (associated with models $\mathrm{H}$ and $\mathrm{P}$ of FC09, respectively) in the $\mathrm{PK}-010$ images, within the standard deviation of measured values. In particular, Fig. 10 shows that computations using the FAL3-F model reproduce the contrast measured for plage features in all the $P K$ images, within the standard 
A\&A 523, A55 (2010)

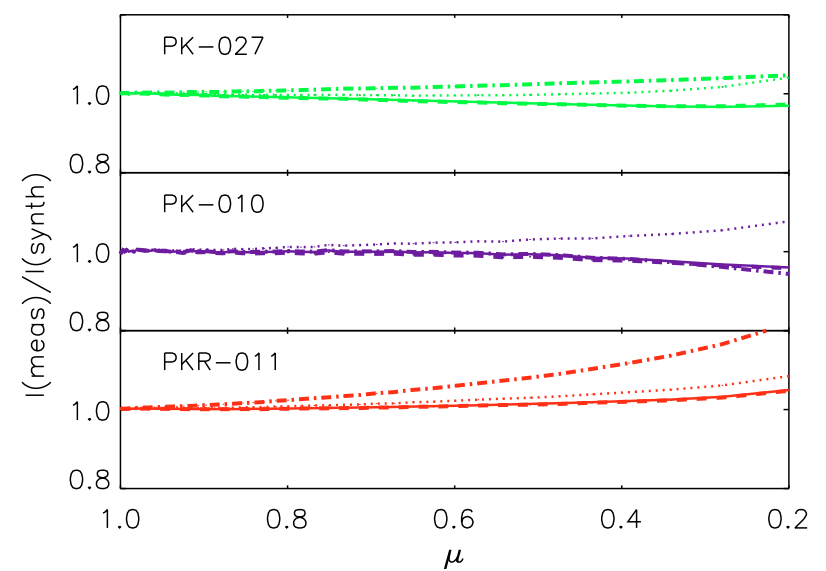

Fig. 9. Ratio of the median CLV measured for quiet Sun regions in PK-027 (top panel), PK-010 (middle panel), and PKR-011 (bottom panel) images to the CLV derived from the synthesis performed with PRD using various atmosphere models. Dotted, solid, dot-dashed, and dashed lines show results derived from the quiet Sun models presented by Fontenla et al. (2009), Fontenla et al. (2006), Vernazza et al. (1981), and Fontenla et al. (1993), respectively.

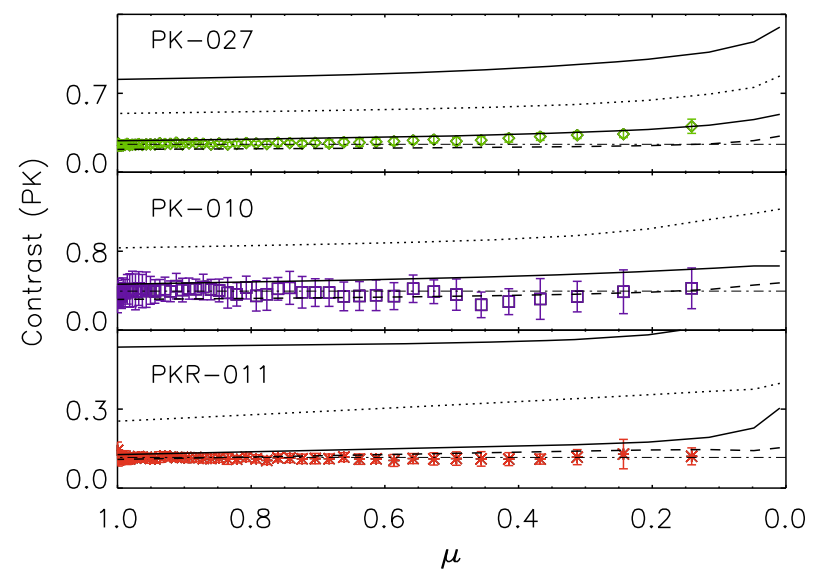

Fig. 10. CLV of median contrast values measured (symbols) for plage regions in $P K-027$ (top panel), $P K-010$ (middle panel), and PKR-011 (bottom panel) images. For each bandpass, the dot-dashed line indicates the average of values measured at disk positions $\mu \geq 0.9$, while dotted, dashed, solid, and triple-dot-dashed lines show the respective CLV derived from calculations with PRD for models $\mathrm{H}$ and $\mathrm{F}$ by Fontenla et al. (2009), and for models F and P by Fontenla et al. (1993), respectively. For $P K-010$, synthesis results for model P of Fontenla et al. (1993) lie outside the plotted range.

deviation of measured values over many of the disk positions analysed. Figure 10 also shows that the same measurements are reproduced by the computations using model F of FC09 more closely than those derived from model $\mathrm{H}$ of the same set, which implies that the correspondence between models and associated features could be improved yet further.

These findings both confirm and extend the results presented by Grigoryeva et al. (2009). These authors compared measurements from spectroscopic observations of QS taken in the spectral range containing the $\mathrm{Ca}$ II $\mathrm{H}$ and $\mathrm{K}$ lines, with computations performed with $\mathrm{RH}$ and PRD using various atmosphere models. In particular, they analysed the VAL3-C and FAL3-C models, as well as the QS model presented by Fontenla et al. (2007). Grigoryeva et al. (2009) found that this latter model, which is quite similar to model B of the FC09 set utilized here, most closely reproduces their intensity CLV measurements in the Ca II K line wings, while at the line core the synthesis results for this model are in poorer agreement with measurements than results for earlier models. We note, however, that our study differs from that carried out by Grigoryeva et al. (2009) in several aspects. In particular, we analysed observations of various solar features, mostly observed in the Ca II K line core, while the other authors restricted their study to QS mainly observed in the line wings. Moreover, we analysed the most recent set of atmosphere models presented in the literature, in addition to the earlier series, while only the latter were considered by Grigoryeva et al. (2009). Finally, we utilized both the PRD and CRD approximations of the spectral synthesis. This allowed us to investigate the effects of code approximations and adopted models.

Discussing the results obtained, we also consider that the $P K$ images are contaminated by low levels of straylight. By checking the falloff of intensity just outside the solar limb, we found that the images analysed in this study show the same contamination level as that estimated by Criscuoli \& Ermolli (2008) in Ca II K observations from the Rome-PSPT. In particular, we found that the ratio of azimuthally averaged intensity values measured at 1.2 solar-disk radii to the intensity measured at disk center is $0.0167 \pm 0.0005,0.0150 \pm 0.0005$, and $0.0120 \pm 0.0005$ for $P K-010, P K-027$, and $P K R-011$ respectively. Therefore, following Criscuoli \& Ermolli (2008) we expect an increase of the order of $30 \%$ in feature contrasts, if they are measured in $P K$ images restored for straylight degradation.

To evaluate the effects of straylight degradation on the comparisons presented above, we developed two test analyses. Firstly, we studied straylight degradation effects on synthetic solar images. In particular, we applied a model point spread function (PSF) to synthetic images derived from our calculations with RH and the FC09 models. We measured the decrease in the intensity and contrast values derived from these synthetic images, hereafter synthetic atmospheres. This provided a rough indication of the influence of straylight contamination on measured contrast values, by avoiding photometric uncertainties introduced by the iterative process of image-restoring methods. We also repeated our measurements for all the features considered in this study in $P K$ images restored from straylight degradation.

The details of the test analyses we performed are given in the appendix. To summarize, we found that the convolution of the model PSF with homogeneous atmospheres, i.e., synthetic atmospheres composed of a single feature at all disk positions, only leads to a significant decrease in intensity and contrast values modeled for the brightest features close to the solar limb. On the other hand, convolution of inhomogeneous atmospheres with the same PSF, i.e., synthetic atmospheres composed of quiet Sun and a number of bright features at various $\mu$ positions, leads to a significant decrease in contrast values estimated for all the features and disk positions. In particular, convolution of the PSF with synthetic atmospheres composed of 10 small features, each covering 0.02 solar-disk radii, on average, leads to a decrease by nearly a factor two in the modeled contrast values. This value is similar to the mean discrepancy between measured and modeled results for the various bright features presented in Sect. 4. In agreement, image restoration from straylight degradation leads to an average increase of $\approx 18 \%$ over all the solar features and a slightly closer agreement between measured and modeled values. Model predictions and contrast values measured in restored images at disk positions $\mu \geq 0.2$ differ by a factor ranging from 0.9 to 2.7 (mean is $\approx 1.6$ ), depending on the solar feature and filter bandpass, this compared to a factor of from 1.1 to 3.1 (mean of $\approx 1.9$ ) for un-restored images. 
While the results of our test analyses indicate that modeled and measured values are in slightly closer agreement after taking into account the effects of straylight contamination, the model predictions are still approximately a factor 1.45 higher than measurements obtained for all the solar features at disk center in restored images.

\section{Conclusions}

We have presented a study of the radiative emission of solar features across the $\mathrm{Ca}$ II $\mathrm{K}$ spectral range. We have analysed moderate-resolution PSPT observations taken with interference filters that sample the Ca II K range with different bandpasses. We have compared the results of CLV measurements for various disk features with the outputs of spectral synthesis performed with a well-validated radiative code and the most recent set of semi-empirical atmosphere models presented in the literature, as well as with earlier similar models. This led to the following results:

(1) In general agreement with results presented in the literature, we found that the CLV of intensity values measured in the Ca II K range for quiet Sun regions depends on both the filter bandpass and image quality. However, the measured dependence is quite small. The quiet Sun's CLV obtained in this study from analysis of the $P K$ images is in striking agreement with published results from observations taken with spectrographs.

(2) The intensity response function for temperature perturbations derived for all the filters considered in this study depends only slightly on the filter bandpass and reference atmosphere; the most sensitive to higher atmospheric layers are the ones derived for the filters with the narrower-band and the reference atmosphere of a bright plage (model P). This model, however, differs from the other models adopted in this study, not only in terms of its higher chromospheric temperature, but also its smaller value of micro-turbulent velocity. We note that even for the narrowest filter, $P K-010$, $58-82 \%$ of the contribution at disk center is from atmosphere heights below $500 \mathrm{~km}$, with the fraction being lower for brighter features, while the same quantity evaluated for $P K-027$, which has a bandwidth similar to that of the SOT/BFI Ca II H filter onboard Hinode, is 84-94\%.

(3) RH calculations with the CRD approximation of the quiet Sun atmosphere (model B) of Fontenla et al. (2009), on average, reproduce measurement results of intensity CLV for quiet Sun regions slightly more closely than PRD computations. However, RH calculations with PRD for earlier models of the quiet Sun atmosphere reproduce measured quantities with a similar accuracy.

(4) The median contrast values measured for most identified features, bandpasses, and disk positions are, on average, a factor $\approx 2$ lower than the contrast values derived from $\mathrm{RH}$ for the reference models considered in this study. Computations in PRD display a closer agreement with the data, which, however, is still unsatisfactory. Calculations for older sets of bright feature models (Vernazza et al. 1981; Fontenla et al. 1993, 2006) fit measurements from plage and bright plage regions as well as those derived from the corresponding new atmosphere models.

(5) For most of the identified features, disk positions, and filter bandpasses analysed in this study, the maximum contrast values measured in $P K$ images are in general closer agreement with model predictions than for median contrast measurements.

(6) The mean discrepancy between modeled and measured values reduces, on average, by about $12 \%$ after taking into account straylight degradation effects on PSPT observations.

In conclusion, the success of CRD computations of the new atmospheres in reproducing some of the quantities analysed in this study does not prove conclusively that the radiative code used in this approximation or the adopted models realistically reproduce the observed quantities. However, it supports the combined use of this formalism and models, as carried out for the spectral modeling of disk features over wide wavelength ranges (e.g. Fontenla et al. 2009). On the other hand, the synthesis performed with PRD, on average, produces radiative estimates that are in closer agreement with measurements achieved in this study from bright disk features than those obtained with the less accurate CRD computation, in particular for the observations taken at the line core. This applies equally-well to synthesis results derived using either the most recent or the earlier atmosphere models, thus supporting simulations performed with PRD on earlier atmosphere sets for diagnostics across narrow spectral ranges of the Ca II K line (e.g. Reardon et al. 2009; Pietarila et al. 2009). The results derived from our investigation also indicate that the neglect of PRD, to some extent, can be compensated for by a change in the reference models. This is very similar to the ambiguous situation reported by Rutten \& Kostik (1982) and Shchukina \& Trujillo Bueno (2001) in the modeling of Fe I lines in the photosphere, where LTE and NLTE calculations can equally well fit the line profiles, as long as the temperature gradient in the atmosphere is adjusted separately in each case.

Acknowledgements. We are grateful to Juan Fontenla for the helpful and fruitful discussions. We also thank the International Space Science Institute (Bern) for giving us the opportunity to discuss this work with the international team on "Interpretation and modeling of SSI measurements". This work was supported by the Istituto Nazionale di Astrofisica (INAF), the Agenzia Spaziale Italiana, grant ASI/ESS I/915/01510710, sub-task 2210, and by the WCU grant No. R31-10016 funded by the Korean Ministry of Education, Science and Technology.

\section{References}

Carlsson, M., \& Stein, R. F. 1995, ApJ, 440, L29

Carlsson, M., \& Stein, R. F. 1997, ApJ, 481, 500

Cauzzi, G., Reardon, K. P., Uitenbroek, H., et al. 2008, A\&A, 480, 515

Criscuoli, S., \& Ermolli, I. 2008, A\&A, 484, 591

Criscuoli, S., Rast, M. P., Ermolli, I., \& Centrone, M. 2007, A\&A, 461, 331

Coulter, R. L., \& Kuhn, J. F. 1994, ASP Conf. Ser., 68, 37

Delbouille, L. Roland, G., \& Neven, L. 1973, Photometric Atlas of the Solar Spectrum from $\lambda 3000$ to $\lambda 10000$, Liège, Université de Liège, Istitut d'Astrophysique

Domingo, V., Ermolli, I., Fox, P., et al. 2009, Space Sci. Rev., 145, 337

Ermolli, I., Fofi, M., Bernacchia, C., et al. 1998, Sol. Phys., 177, 1

Ermolli, I., Criscuoli, S., Centrone, M., Giorgi, F., \& Penza, V. 2007, A\&A, 465, 305

Ermolli, I., Solanki, S. K., Tlatov, A., et al. 2009, ApJ, 698, 1000

Fontenla, J. M., Avrett, E., \& Loeser, R. 1991, ApJ, 377, 712

Fontenla, J. M., Avrett, E. H., \& Loeser, R. 1993, ApJ, 406, 319 (FAL3)

Fontenla, J. M., Avrett, E., Thuillier, G., \& Harder, J. 2006, ApJ, 639, 441 (FA06)

Fontenla, J. M., Balasubramaniam, K. S., \& Harder, J. 2007, ApJ, 667, 1243

Fontenla, J. M., Curdt, W., Haberreiter, M., Harder, J., \& Tian, H. 2009, ApJ, 707, 482 (FC09)

Goldbaum, N., Rast, M. P., Ermolli, I., Summer Sands, J., \& Berrilli, F. 2009, ApJ, 707, 67

Grigoryeva, S. A., Teplitskaya, R. B., \& Ozhogina, O. A. 2009, Astron. Reports, 53, 569

Harder, J. W., Fontenla, J. M., Pilewskie, P., Richard, E. C., \& Woods, Th. N. 2009, Geophys. Res. Lett., 36, 7, L07801 
Hansteen, V., de Pontieu, B., Carlsson, M., et al. 2007, Publ. Astron. Soc. Japan, 59, S699

Kentischer, T. J., Bethge, Ch., Elmore, D. F., et al. 2008, Ground-based and Airborne Instrumentation for Astronomy II, ed. I. S. McLean, \& M. M. Casali, Proc. SPIE, 7014, 701413

Krivova, N. A., Solanki, S. K., \& Wenzler, T. 2009, Geophys. Res. Lett., 36, 30, L20101

Lawrence, J. K., \& Cadavid, A. C. 2010, Sol. Phys., 261, 35

Leenaarts, J., Carlsson, M., Hansteen, V., \& Rouppe van der Voort, L. 2009, ApJ, 694, L128

Livingston, W., \& Sheeley, N. R. Jr. 2008, ApJ, 672, 1228

Maltby, P., Avrett, E. H., Carlsson, M., et al. 1986, ApJ, 306, 284

Meléndez, M., Bautista, M. A., \& Badnell, N. R. 2007, A\&A, 469, 1203

Nesme-Ribes, E., Meunier, N., \& Collin, B. 1996, A\&A, 308, 2213

Pietarila, A., Hirzberger, J., Zakharov, V., \& Solanki, S. K. 2009, A\&A, 502, 647

Rast, M. P., Fox, P. A., Lin, H., et al. 1999, Nature, 401, 6754, 678

Rast, M. P., Ortiz, A., \& Meisner, R. W. 2008, ApJ, 673, 1209

Reardon, K. P., Uitenbroek, H., \& Cauzzi, G. 2009, A\&A, 500, 1239

Rezaei, R., Bruls, J. H. M. J., Schmidt, W., et al. 2008, A\&A, 484, 503

Rutten, R. J. 2007, in The Physics of Chromospheric Plasmas, ed. P. Heinzel, I. Dorotovic, \& R. J. Rutten, ASP Conf. Ser., 368, 27

Rutten, R. J., \& Kostik, H. 1982, A\&A, 115, 104
Shchukina, N., \& Trujillo Bueno, J. 2001, ApJ, 550, 970

Scharmer, G. B., Bjelksjo, K., Korhonen, T. K., et al. 2003, Innovative Telescopes and Instrumentation for Solar Astrophysics, ed. S. Keil, \& S. Avakyan, Proc. SPIE, 4853, 341

Scharmer, G. B., Narayan, G., Hillberg, T., et al. 2008, ApJ, 689, L69

Shine, R. A., \& Linsky, J. L. 1974, Sol. Phys., 39, 49

Shine, R. A., Milkey, R. W., \& Mihalas, D. 1975, ApJ, 201, 222

Tritschler, A., Schmidt, W., Uitenbroek, H., \& Wedemeyer-Böhm, S. 2007, A\&A, 462, 303

Tsuneta, S., Suematsu, Y., Ichimoto, K., et al. 2008, Sol. Phys., 249, 167

Uitenbroek, H. 1989, A\&A, 213, 360

Uitenbroek, H. 2001, ApJ, 557, 389

Uitenbroek, H. 2002, ApJ, 565, 1312

Uitenbroek, H. 2006, ASP Conf. Ser., 354, 313

Vernazza, J. E., Avrett, E. H., \& Loeser, R. 1981, ApJS, 45, 635 (VAL3)

Wedemeyer-Böhm, S., Freytag, B., Steffen, M., Ludwig, H. G., \& Holweger, H. 2004, A\&A, 414, 1121

White, O. R., \& Suemoto, Z. 1968, Sol. Phys., 3, 523

Wittmann, A. 1976, A\&A, 48, 121

Wöger, F., Wedemeyer-Böhm, S., Schmidt, W., \& von der Lühe, O. 2006, A\&A, 459, L9

Zirker, J. B. 1968, Sol. Phys., 3, 164

Pages 11 to 14 are available in the electronic edition of the journal at http://www. aanda. org 


\section{Appendix A: Straylight contamination}

To evaluate the effects of straylight degradation on the comparisons between modeled and measured quantities presented in Sect. 4, we developed two test analyses. Firstly, we measured the effects of the straylight contamination induced by a model point spread function (PSF) on synthetic solar images. The PSF used in our analysis follows Criscuoli \& Ermolli (2008); it includes one Lorentzian and three Gaussian components, which are defined by seven parameters. We fixed six parameters of the PSF components derived by Criscuoli \& Ermolli (2008), while setting the remaining parameter to reproduce the average value of the straylight level measured in $P K$ images at 1.2 solar-disk radii. This parameter describes the weight of the Lorentzian relative to the Gaussian components.

Figure A.1 shows the model PSF utilized in our analysis. We convolved synthetic images constructed from intensities calculated with the one-dimensional FC09 models for the various disk features and bandpasses analysed in our study with the same model PSF. We constructed two sets of synthetic atmospheres. The first set is composed of homogeneous atmospheres. The second set contains inhomogeneous atmospheres with two components, each atmosphere displaying quiet Sun and a number of features of the same type at various $\mu$ positions. We describe in the following the results obtained by assuming 10 features, each covering 0.02 solar-disk radii at all $\mu$ positions, i.e. foreshortening was not taken into account. For the various FC09 models at the $P K-010$ bandpass, Figs. A.2 and A.3 show the intensity values computed at various $\mu$ positions in the original synthetic atmospheres and the atmospheres convolved by the model PSF.

We found that the convolution of the model PSF with synthetic homogeneous atmospheres leads to a significant decrease only in the intensity and contrast values modeled for the brightest disk features close to the solar limb. Figure A.4 shows that the discrepancy between measured and modeled CLV for QS increases when moving toward the limb if straylight contamination of $P K$ images is taken into account, except when comparing the CLV measured for $P K-027$ and the one derived from CRD computations. Moreover, we found that $P K-027$ measurements are in close agreement with the CLV obtained from the inhomogeneous atmospheres containing network features after their straylight contamination.

On the other hand, we found that the convolution of synthetic inhomogeneous atmospheres with the same PSF leads to a significant decrease in contrast values estimated for all the features and disk positions. This is illustrated by Figs. A.5 and A.6, which compare the results presented in Sect. 4, and the CLV derived from the convolution of the synthetic atmospheres with the model PSF. The contrast values estimated in the synthetic atmospheres convolved by the PSF are by up to nearly a factor two lower than those obtained in the original RH computations. Therefore, these results suggest a closer general agreement between median contrast values measured in $P K$ and the contrast values derived from the RH synthesis than presented in Sect. 4 , if straylight contamination of small-scale disk features is taken into account.

Our second test analysis was performed on $P K$ images restored for straylight contamination by the deconvolution of original images with a model PSF derived from the analysed data. We applied the method and algorithm developed by Criscuoli \& Ermolli (2008) to the whole sample of $P K$ good images analysed in Sect. 4.

Figures A.7 and A.8 compare model results presented in Sect. 4 and the CLV of contrast values measured for the various

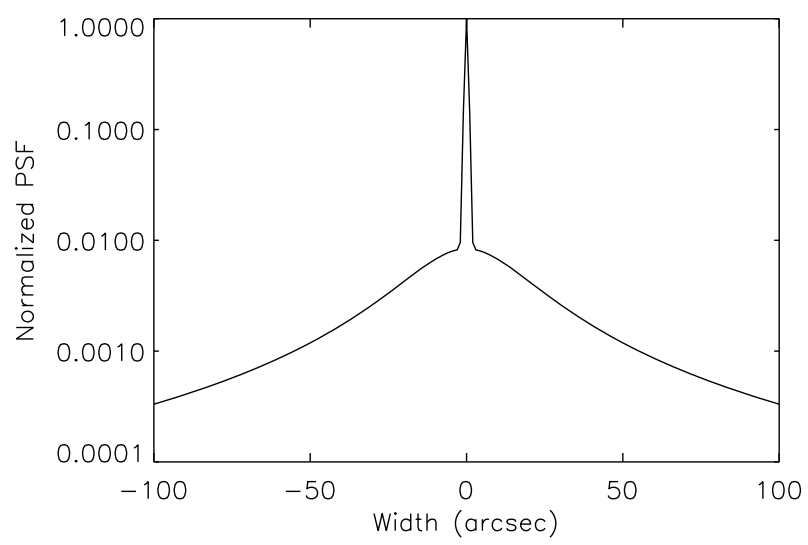

Fig. A.1. The model PSF used to evaluate effects of straylight degradation on PK images follows Criscuoli \& Ermolli (2008). It includes three Gaussians and one Lorentzian component.

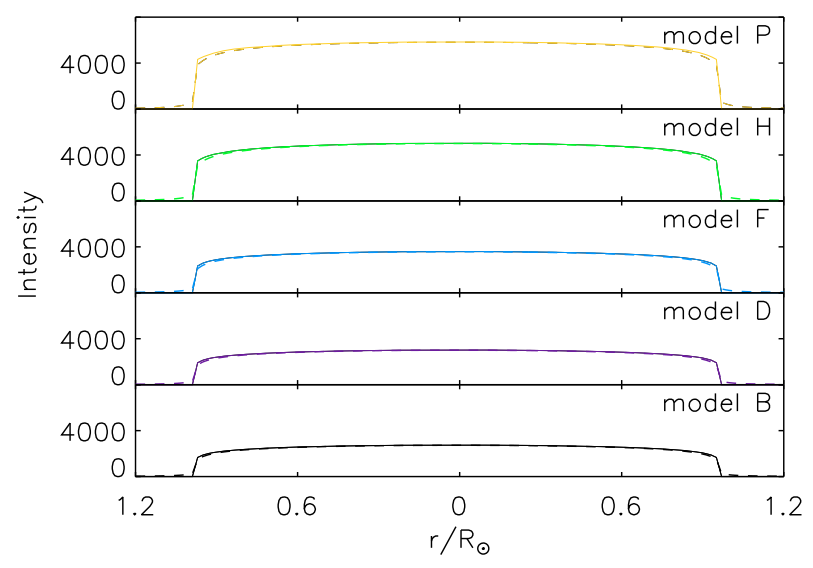

Fig. A.2. Profiles of intensity values in the set of synthetic homogeneous atmospheres (solid lines) computed for the various disk features and $P K$-010 bandpass. In the various sub-panels, the different atmospheres are identified by colors and labels: yellow, green, blue, violet, and black show bright plage, plage, enhanced network, network, and quiet Sun atmospheres, associated with models P to B, respectively. Distance from disk center is given in disk radius units. The dashed lines show the profiles of intensity values in the various atmospheres after their convolution by the model PSF.

solar features on images corrected for straylight degradation. We found that image restoration leads to a slight increase in the average contrast values measured for all solar features with respect to the values derived from un-restored images, in particular for the features identified in $P K-027$ and $P K-010$ images. In addition, we found an increase in the maximum contrast values measured for all the features, filter bandpasses, and disk positions, in particular for the network features in $P K$-027 images. We also found slightly closer agreement between model predictions and contrast values measured in restored images than derived from un-restored images. We found that model predictions and contrast values measured at disk center $(\mu \geq 0.9)$ in restored images indeed differ by a factor ranging from 0.81 to 2.17 , depending on the solar feature and filter bandpass, while the same quantity evaluated on un-restored images ranges from 0.98 to 2.58 . Modeled and measured values differ, on average, by a factor of 1.45 after taking into account straylight effects, compared to a factor of 1.71 for measurements in un-restored images at the same disk positions. 


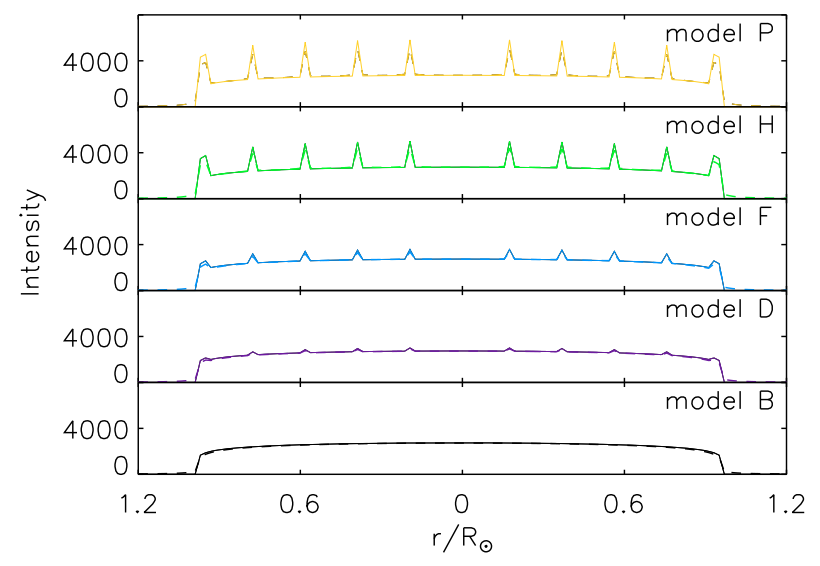

Fig. A.3. Profiles of intensity values in the set of synthetic inhomogeneous atmospheres (solid lines) computed for the various disk features and $P K-010$ bandpass. Legend as given in Fig. A.2. The dashed lines show the profiles of intensity values in the various atmospheres after their convolution by the model PSF. Distance from disk center is given in disk radius units.

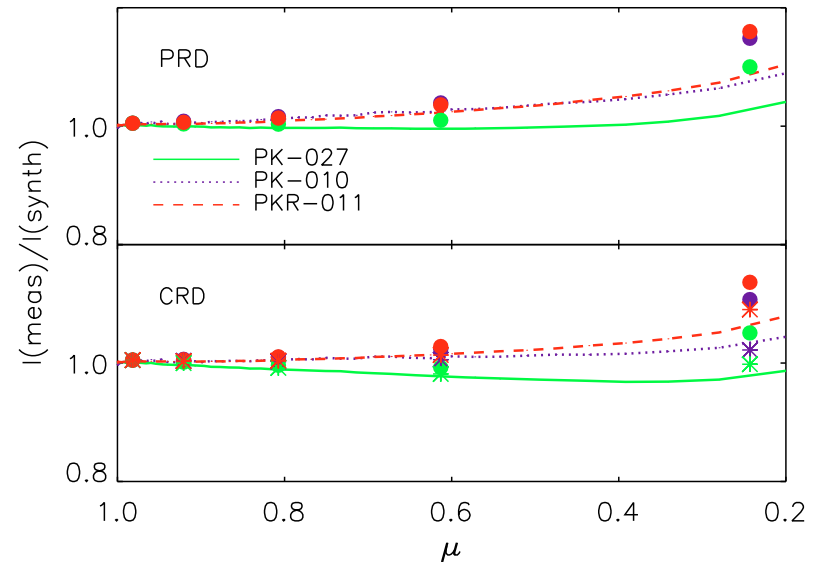

Fig. A.4. Ratio of the median of quiet Sun limb darkening measured in PSPT images to the quiet Sun limb darkening derived from the synthesis performed for the $P K-027$ (green, solid line), $P K$ - 010 (violet, dotted line), and $P K R-011$ (red, dashed line) filters by assuming either the PRD (top panel) or CRD (bottom panel) approximation. The lines indicate the results obtained by taking into account the CLV derived from the synthesis, while circles show the results derived by convolving the synthetic homogeneous quiet Sun atmosphere (associated with model B) by the model PSF. Asterisks show the ratio of the CLVs measured in $P K$ to the ones derived from contaminated inhomogeneous atmosphere composed of quiet Sun and network small features, associated with models B and D, respectively.
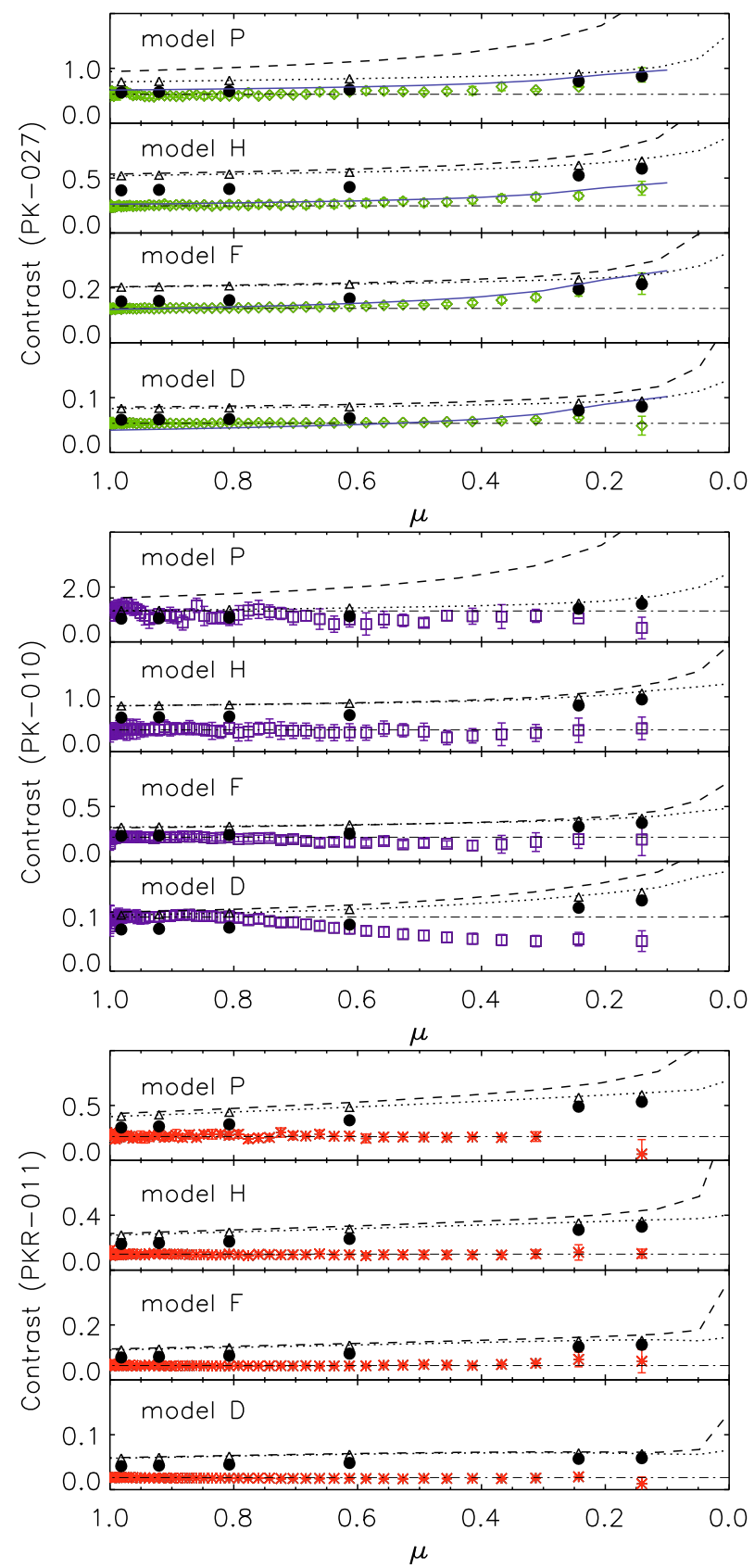

Fig. A.5. CLV of median contrast values measured (symbols) for various disk features identified in $P K-027$ (top panel), $P K$-010 (middle panel), and PKR-011 (bottom panel) images un-restored for straylight degradation. For each bandpass, the various sub-panels show measurement results for solar features ordered by decreasing contrast, i.e. from the top panel results for bright plage, plage, enhanced network, and network regions, respectively. The error bars represent the standard deviation of measurements. For each disk feature and bandpass, the dotdashed line indicates the average of values measured at disk positions $\mu \geq 0.9$, while dotted and dashed lines show the respective CLV derived from RH calculations with PRD and CRD and the reference model corresponding to the given feature, also indicated in the legend. Triangles and circles indicate the results obtained after convolution of the synthetic atmospheres by the model PSF, for homogeneous and inhomogeneous atmospheres, respectively. In the top panel, the solid blue lines show the threshold values applied to the feature identification. 
I. Ermolli et al.: Radiative emission of solar features in the Ca II K line
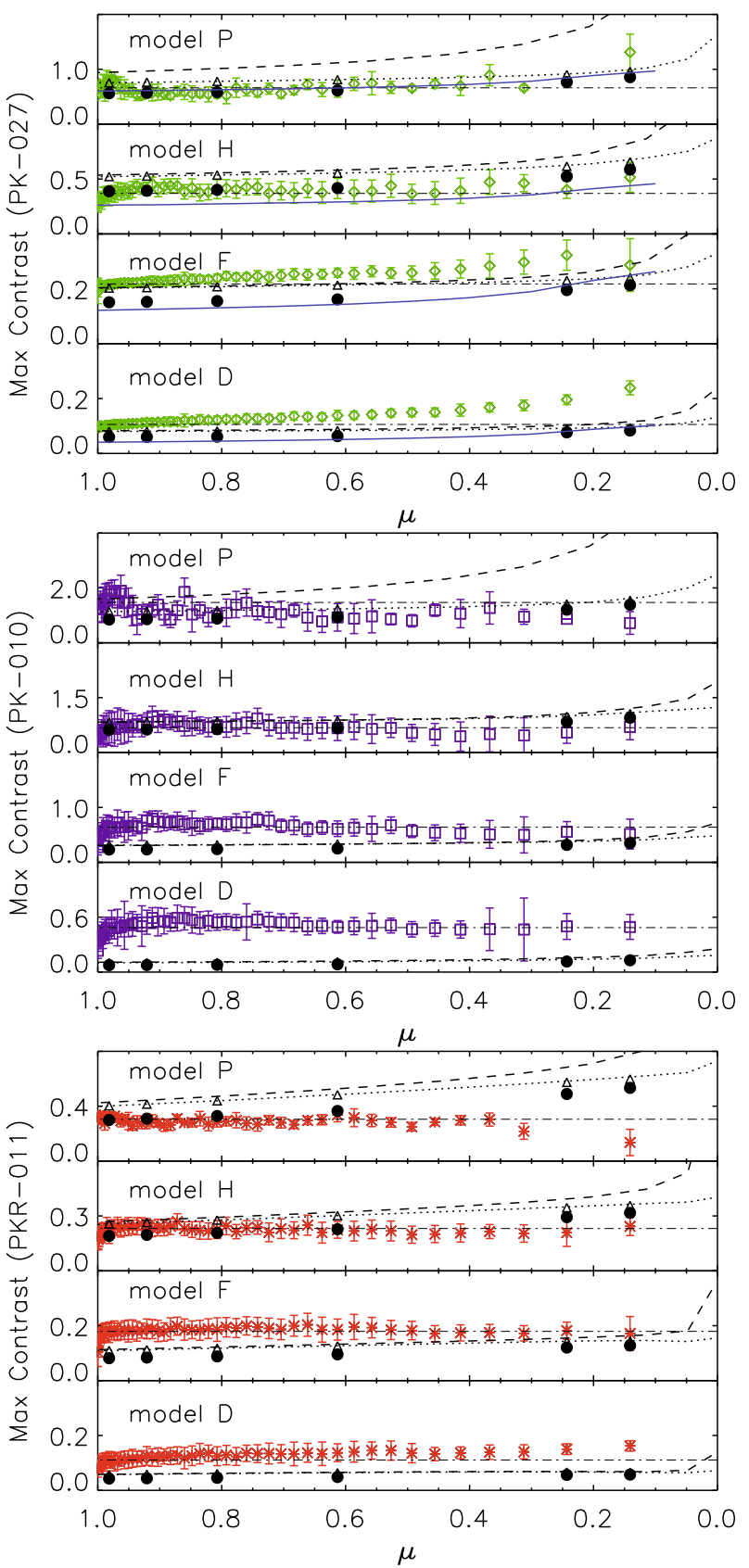

Fig. A.6. CLV of maximum contrast values measured (symbols) for various disk features (model P to model D) in $P K-027$ (top panel), PK-010 (middle panel), and PKR-011 (bottom panel) images un-restored for straylight degradation. Legend as given in Fig. A.5.
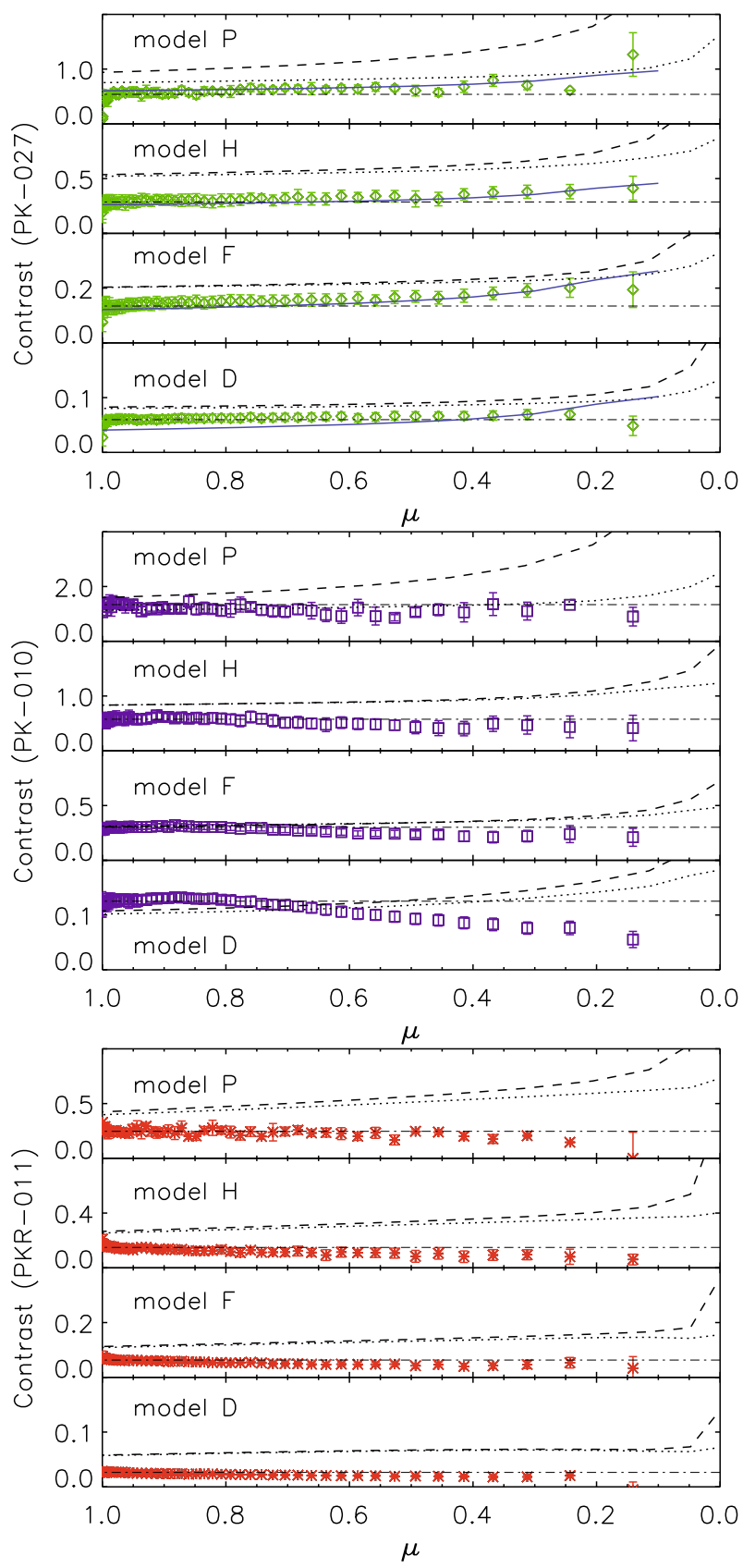

Fig. A.7. CLV of median contrast values measured (symbols) for various disk features identified in $P K-027$ (top panel), $P K$-010 (middle panel), and PKR-011 (bottom panel) images restored from straylight degradation. For each bandpass, the various sub-panels show measurement results for solar features ordered by decreasing contrast, i.e. from the top panel results for bright plage, plage, enhanced network, and network regions, respectively. The error bars represent the standard deviation of measurements. For each disk feature and bandpass, the dotdashed line indicates the average of values measured at disk positions $\mu \geq 0.9$, while dotted and dashed lines show the respective CLV derived from RH calculations with PRD and CRD and the reference model corresponding to the given feature, as also indicated in the legend. In the top panel, the solid blue lines show the threshold values applied to the feature identification. 
A\&A 523, A55 (2010)
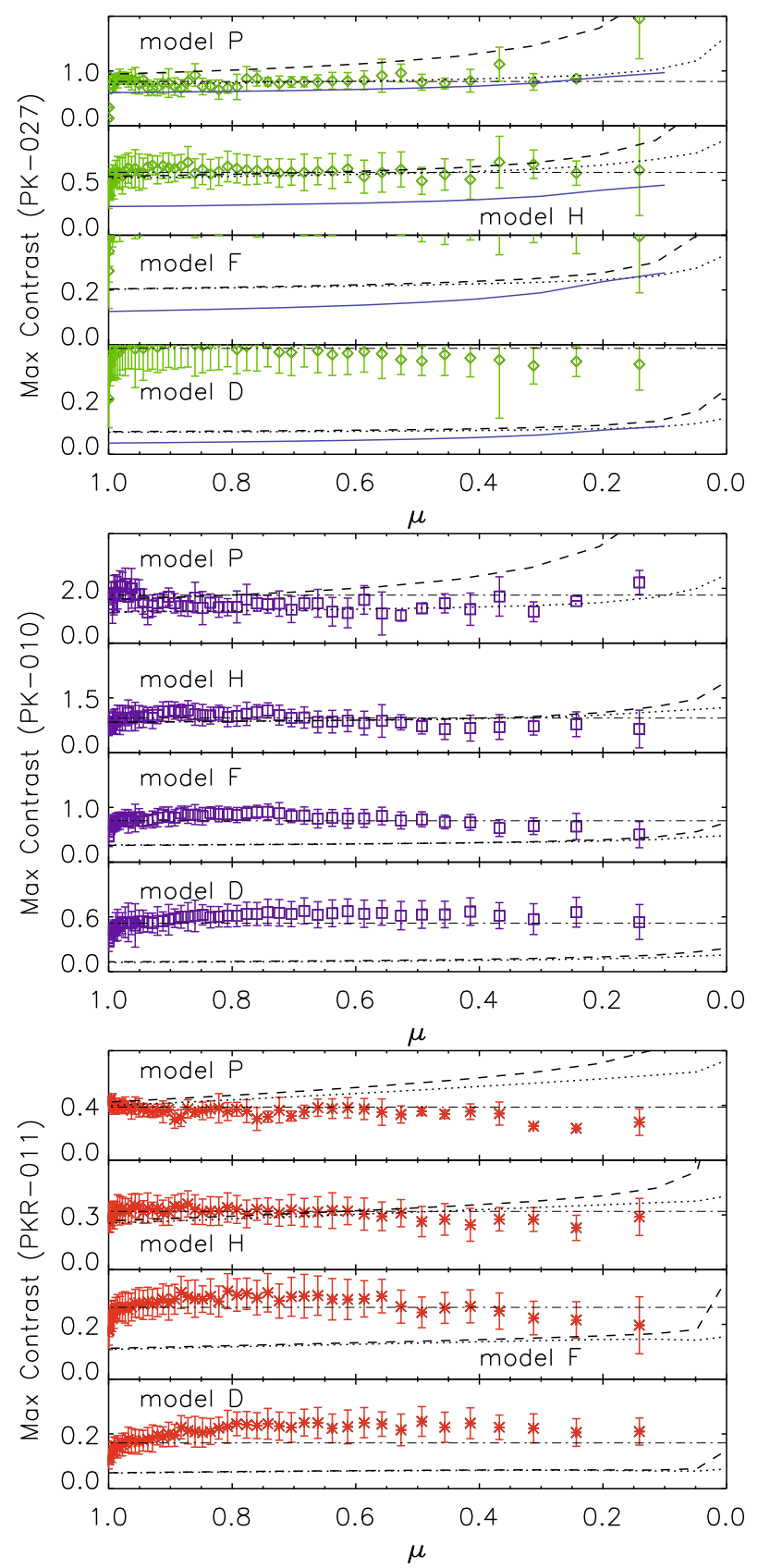

Fig. A.8. CLV of maximum contrast values measured (symbols) for various disk features (model $\mathrm{P}$ to model D) in $P K-027$ (top panel), PK-010 (middle panel), and PKR-011 (bottom panel) images restored from straylight degradation. For $P K-027$, measurement results for enhanced network and network features (associated with model F and D of Fontenla et al. (2009), respectively) lie outside the plotted range, that is the range utilized for results from un-restored images. Legend as given in Fig. A.7. 